\title{
A Multi-touch Approach to Control MRI Scans: A User-Centered Study Report
}

\author{
Tulio de Souza Alcantara, Pierre Bastianelli, Jennifer Ferreira, Frank Maurer \\ Department of Computer Science, University of Calgary \\ $\{$ tuliosouza,pbastia\}@gmail.com \\ \{jen.ferreira,frank.maurer\}@ucalgary.ca
}

\begin{abstract}
This paper reports on a study investigating the usability challenges faced by users of Magnetic Resonance Imaging (MRI) tools. In order to understand these problems, observation, shadowing and interviews were conducted with MRI scan users at two centers. After analyzing the collected data, low-fidelity prototypes were created and evaluated. We addressed the usability issues found by proposing a userfriendly and efficient high-fidelity prototype that replaces keyboard and mouse with two multi-touch screens.
\end{abstract}

Keywords-interactive surfaces, MRI, UCD, usability, GUI, multi-touch

\section{INTRODUCTION}

Magnetic Resonance Imaging (MRI) machines are used to obtain pictures from the inside of biological tissue, in a non-intrusive, non-destructive way.

These machines have continually improved in the last decades due to better magnetic field intensity, precision of the equipment, computing power and image processing algorithms.

As observed by the authors, operating these machines is a difficult task, requiring a good understanding of the underlying MRI physics and experience with the peculiarities of the complex, machine-specific software. Not only MRI machines but, as reported by Muto and Israelski in 2007, the usability of other medical devices can benefit from the advances of new technologies, such as large display screens and touchscreens [15].

This is why a fast, efficient and simple way of setting up the machine according to simple parameters is required, in order for medical practitioners to use basic functionalities of MRI imaging, without the need for training with complex software or extensive manuals.

The main goal of this study is to understand the usability challenges and finding new ways to improve the usability and broaden the target user group for these machines, using interactive surfaces or tabletops such as Evoluce table [11].

The technologies allowing the production of interactive surfaces are constantly improving and operating systems and Software Development Kits (SDKs) are starting to provide native support for these technologies. Interactive surfaces also allow the use of more natural gestures and direct manipulation of the whole GUI [14]. The benefits expected from such an interface are a better communication between the MRI physicist and the scientist for whom the experiment is run, and the ability for non-experts to run simple imaging experiments or to gather data from it.

In order to design a product that better suits the needs of the MRI machine users we followed an iterative Usercentered design (UCD) method [9].
A business analysis was conducted by shadowing MRI experiments, performing observation and interview sessions [3], and production of work scenarios. This was then followed by brainstorming sessions, leading to a first set of low-fidelity prototypes. These prototypes were evaluated for usability problems and design flaws.

Following the evaluations, a second iteration of the process was started: brainstorming sessions were conducted again, leading to a high-fidelity prototype using two multitouch screens which was representative for what a final interface for the MRI machine could be.

The evaluation sessions of both prototypes used the Wizard of $\mathrm{Oz}$ concept [1] and showed an excellent acceptance by the participants of the study, particularly with less experienced MRI machine users. As a result of this work, tabletops and multi-touch gestures associated with a well-designed interface can be considered as a good answer to the complexity of current MRI interfaces.

The next section of this paper discusses related work. This is followed by a study overview. The fourth section explains the usability study with its steps in a more detailed way. The fifth section shows the evaluation results. The last section shows a conclusion and future work to this study.

\section{RELATED WORK}

The current literature contains several reports on research with MRI machines and especially image treatment $[5,12,13]$. These reports focus on functionality rather than usability, producing software that, as observed by the authors, has many complex functionalities and applications but due to its usability problems, impedes its use.

Several studies pointed out usability issues within MRI software: Gallo et al. addressed usability issues by proposing a tool to interact with $3 \mathrm{D}$ volumetric medical data [2]. Also in medical images, in 2009 Skounakis et al. proposed an easy-to-use annotation platform for identification of tumors in medical images [6]. Ure et al. published a study that outlines the human and technical challenges to design a portal for neuroscience, MRI and Grid computing [7]. These solutions targeted specific issues in MRI scans (interacting with volumetric data, image annotation) or propose a portal for diverse areas whereas the present study addresses the usability problem in the context of the whole workflow of MRI.

Rogers and Lindley explored the use of vertical and horizontal multi-touch tables [4]. They found that both orientations of displays encourage groups to work together and that vertical displays are better at providing a shared surface for groups and annotating of information. In a similar fashion, the high-fidelity prototypes in this paper use two screens: one horizontal and one vertical. 


\section{STUDY OVERVIEW}

There were two MRI scan facilities used for this study:

(A) has a 9.4T MRI machine with a rat-sized inmagnet space and

(B) has a $0.2 \mathrm{~T}$, open magnet MRI machine.

The two MRI machines were used in different ways. The machine (A) was used for veterinary research, mostly on mice and rats. It was also used for brain imaging and investigation of contrast agent injections.

MRI (B) was used for two different purposes: imaging of bigger animals (cows, sheep), sometimes even human patients, and research on MRI protocols. As this machine was built entirely by the research center they are a part of, the users have more the profile of MRI researchers instead of radiologists or biologists. The main user of this machine is constantly trying to improve the MRI sequences in order to get better image quality, by manually modifying the code handling the MRI hardware.

The UCD study was conducted using the data collection techniques described by Lethbridge et al. in 2005 [3].

The first observation sessions were conducted with users from location (B) followed by interviews with these users.

After the interviews, a documentation analysis of the operating manual of the MRI machine from location (A) and (B) gave the authors a better understanding of the technical aspects of MRI control software [3].

Based on the data collected in the previous steps, lowfidelity prototypes were created using Active Story Touch (AST) [10] a low-fidelity prototyping tool for touch devices, allowing dynamic navigation through the prototypes. These prototypes were then evaluated with users from (A) and (B).

During the evaluation of the low-fidelity prototypes, users were encouraged to make comments and give their impressions of the low-fidelity prototypes, according to the think-aloud protocol [3].

Finally, with the data collected from the evaluation of the low-fidelity prototypes, the authors created high-fidelity prototypes using two multi-touch interaction displays, one horizontal and one vertical.

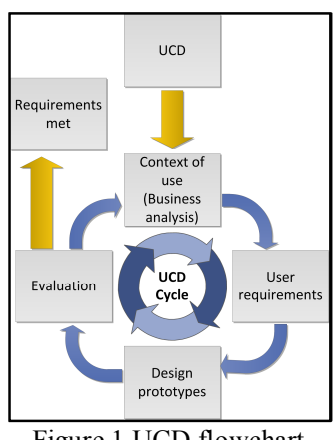

Figure 1 UCD flowchart

The UCD process was applied as following: Business analysis, establishment of the user requirements, prototyping and evaluation, the whole in an iterative manner as shown in the flowchart in Figure 1 [9]. The UCD cycle was repeated until the requirements obtained through the business analysis had been met.

\section{User-CENTERED DESIGN STUDY}

A thorough business analysis of MRI operation was run in order to get a broader picture of the user's work, to identify the tasks the user has to complete in the whole workflow and to understand the problems the user is facing with the current system.

\section{A. Business Analysis}

The business analysis allowed the identification of the users who will use the software, what they will use it for, and under what conditions they will use it. It also allowed the identification of user stories, specifying the multiple functional requirements in each case.

1) Observations and Background Interviews: This step consisted of observing the interactions and the daily work of the users (shadowing) [3] from both locations. In this step the authors gained an understanding of the user's workflow and their use of the MRI machines.

Later in the process, interviews were conducted with users from both locations to clarify assumptions made during the observation sessions.

2) Documentation Review: This step gave a first set of what functionalities a new tool should provide and helped to identify some design flaws in the existing software. The results were shared with the users, which gave them a chance to provide feedback. In this way, the researchers could verify their assumptions.

Having access to two different machines allowed the authors to analyze similarities or differences between the two operating manuals during the documentation review.

The outcome of the documentation review was a list of functionalities that are required for a MRI scan. The identified functionalities were used as the base for the requirements of the software, informed by the results from the observations.

3) Users Definition: Based on the information collected and analyzed at this stage, the authors were able to define the potential users. The following users were identified: engineer, operator (technician), scan browser and medical study contractor.

The engineer works on a research MRI machine; the goal of the machine is not only to provide images but also to support research activity on how to improve MRI machines. The engineer is highly experienced with configuring MRI machines and is knowledgeable about the physics and technology of MRI machines.

The operator ensures that all the images are taken according to what the research protocol specifies. The operator advises scientists on the setup of a research experiment, and helps them to define the appropriate imaging protocol for what they are looking for. The operator is also responsible for the backup of the data collected during experiments and for showing and handing over the resulting images to the scientists. 
The scan browser can be a doctor or a biologist who wants to see an older scan and manipulate the images. Quite frequently, people come directly to the MRI machine in order to see some scans taken before.

The medical study contractor is not a real user of the MRI machine itself, but the contractor for the MRI study. During the observations, it was noticed that the contractor has to ask the MRI operator to perform the scans, according to a defined protocol. The reason why the contractor is not performing the scan is due to the complexity of operation of the machine. If a really simple interface with very few parameters is available, then the need for the MRI operator disappears in that particular case.

\section{B. User Requirements}

Based on the defined users, two kinds of interfaces were considered. The first is a simple, user-friendly interface for occasional users. The second is a more advanced (but still simple, user-friendly) interface for technicians and/or trained scientists. The goal of the following steps in this stage of the UCD process is to obtain a set of requirements.

1) Scenarios: Were defined in order to identify the activities that the tool should cover. Additionally, the scenarios helped the authors in creating the prototypes and motivated the user during the prototype's evaluations, as they could choose among the solutions according to the context. The design scenarios are presented below:

TABLE I. USE CASE SCENARIOS

\begin{tabular}{|c|c|c|}
\hline \multicolumn{2}{|r|}{ Scenario } & Description \\
\hline 1 & $\begin{array}{l}\text { Continuing } \\
\text { an } \\
\text { experiment }\end{array}$ & $\begin{array}{l}\text { A scientist with very little knowledge about MRI } \\
\text { machines and working alone wants to continue an } \\
\text { existing experiment: The subject is fit in the coil, } \\
\text { experiment selected, slices adjusted and scan run. } \\
\text { Calibration is done automatically, as well as taking } \\
\text { the scout pictures for positioning the slices. }\end{array}$ \\
\hline 2 & $\begin{array}{l}\text { Do a one- } \\
\text { shot image }\end{array}$ & $\begin{array}{l}\text { A MRI operator received a command for a special } \\
\text { image and wants to use manual calibration } \\
\text { (frequency adjustment, shimming, and pulse } \\
\text { calibration) to fine-tune the machine. Feedback is } \\
\text { provided by images taken and imaging settings can } \\
\text { also be fine-tuned. It is possible to take a scout } \\
\text { image anytime to see the result. }\end{array}$ \\
\hline 3 & $\begin{array}{l}\text { Multi-user } \\
\text { situation, } \\
\text { MRI } \\
\text { operator } \\
\text { and } \\
\text { scientist }\end{array}$ & $\begin{array}{l}\text { Two users (an operator and a scientist), typically } \\
\text { setting up and starting an experiment or doing } \\
\text { custom imaging for existing scans. While the } \\
\text { operator calibrates the machine and adjusts imaging } \\
\text { parameters, the scientist registers the experiment } \\
\text { and starts selecting the slices for imaging. }\end{array}$ \\
\hline 4 & $\begin{array}{l}\text { Research } \\
\text { on MRI } \\
\text { sequences }\end{array}$ & $\begin{array}{l}\text { A MRI researcher is doing the imaging of objects } \\
\text { significant for living tissue: rubber band, asparagus } \\
\text { etc. The goal is to find out which sequences are the } \\
\text { best for imaging the probe. The MRI researcher } \\
\text { modifies the source code of the sequence files } \\
\text { manually and stores the best sequence and makes it } \\
\text { available for real experiments. }\end{array}$ \\
\hline 5 & $\begin{array}{l}\text { Browse the } \\
\text { scan } \\
\text { database }\end{array}$ & $\begin{array}{l}\text { A user who is looking for a specific scan wants to } \\
\text { browse the database to look for it. With the selected } \\
\text { scan, the user might export the scan images, display } \\
\text { the scan images or display the scan settings }\end{array}$ \\
\hline
\end{tabular}

2) Brainstorming Sessions: The goal here was to have authors and users creating and discussing potential design ideas for the chosen scenarios. Good ideas were then selected and refined, and taken into the low-fidelity prototyping stage.

The authors met with users from location (B) and used a white board express design ideas and discuss them. With the ideas refined by the brainstorming sessions, the authors created the low-fidelity prototypes using AST.

After the brainstorm sessions, it was decided to create a low-fidelity prototype to address the problems in scenarios 1,2 and 5. The users didn't consider scenario 3 as a fundamental need for the interface and scenario 4 was not prioritized because it represents a usage of the MRI machine that is too specific. In order to cover the three selected scenarios, a "wizard" interface was chosen.

\section{Design Prototypes}

To refine the design ideas, the authors decided to carry out this stage of the UCD cycle in two steps: first creating low-fidelity prototypes that allowed the authors to experiment with several design ideas and evaluate them. The second step used the evaluated low-fidelity prototypes to create high-fidelity prototypes that are closer to a final application.

1) Low-Fidelity Prototypes: The current literature shows that low-fidelity prototypes improve the design of touchbased applications for multi-touch surfaces and allows a more user-centered design of applications [8].

AST is a low-fidelity prototyping tool that enables fast sketching of UI design screens and to link those screens together [10], supporting playback with multi-touch gestures. The low-fidelity prototypes were created using the results of the brainstorming sessions. Every different prototype addressed one or more use case scenarios.

Navigation between the different pages of the prototypes was triggered by multi-touch gestures.

2) High-Fidelity Prototypes: The low-fidelity prototypes were used for establishing the design specification of the high-fidelity prototype. All the sketch-like prototypes related to the chosen scenario were gathered, and used as a design base for a high-fidelity implementation, completed by a graphics chart.

Figure 2 shows how a low-fidelity prototype in AST evolved to a high-fidelity prototype after user evaluation.

The high-fidelity prototypes were created using Windows Presentation Foundation (WPF) [16] and were designed to be used with a layout that consisted of two multi-touch screens: one horizontal and one vertical, as shown in Figure 3.

\section{Evaluation}

The low-fidelity prototypes were evaluated on a 12.1 inch multi-touch tablet at the users' workplace at both locations. Evaluation was done twice with a single user, and once with three users, collaborating on the task. 
During the Wizard of $\mathrm{Oz}$ evaluation, one author observed, took notes on the potential mistakes the user made, or on the errors contained in the user interface. The other author guided the user through the interface, in case the prototype was not clear enough, and assumed the role of the wizard.

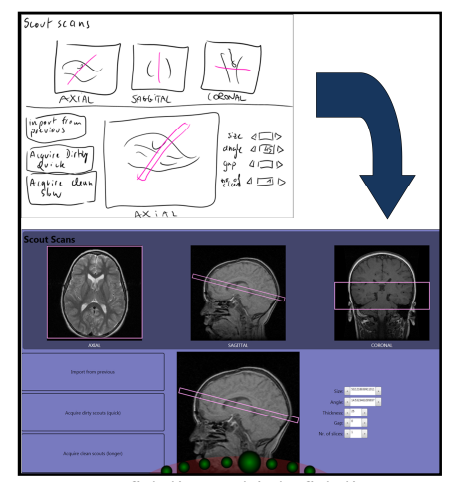

Figure 2: Low-fidelity to high-fidelity prototype

During the evaluations the users were encouraged to express their opinions about the prototypes, according to the think aloud protocol [3].

The limitations of the evaluation of the low-fidelity prototypes are due to the device chosen for the prototypes. In order to run the evaluation in the user's working environment, a portable device was chosen, which made it single-user only and on a 12.1 inch screen. Due to AST's limitation of working with multiple screens, the low-fidelity prototypes needed to be adapted for a single screen. This was made by drawing a horizontal line on the prototypes.

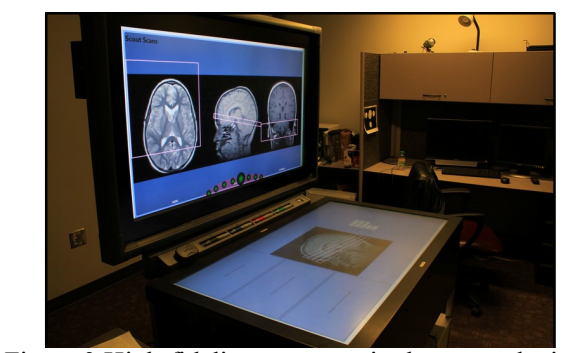

Figure 3 High-fidelity prototype in the target device

The high-fidelity prototypes were evaluated in a layout as shown in Figure 3 and were evaluated by users from location (B) only. The evaluation was done in a similar fashion as for the low-fidelity prototype.

\section{CONCLUSION AND FUTURE WORK}

The high-fidelity prototype interface development will continue until the interface covers all the scenarios identified in the beginning of the design phase.

The high-fidelity prototype presented here is proposed to evaluate usability features and does not contain any functionality. The need for more powerful settings was also identified when the user is experienced with MRI physics and technics which are covered by scenario 4 .
Furthermore, specific care was taken that the results of this study could not only be applied to the particular MRI environment that was observed, but that this study could be helpful for other MRI environments.

This study has all the chances of proving successful: all the positive feedback from the evaluation of the low-fidelity prototypes and from the ongoing evaluation suggests that the use of tabletops and touch-interfaces are actually improving the user experience on the control of MRI machines, and that the interface proposed in this paper will require less cognitive load, which could free up the medical practitioner to focus on important tasks.

\section{REFERENCES}

[1] Akers, D. (2006). Wizard of $\mathrm{Oz}$ for participatory design: inventing a gestural interface for 3D selection of neural pathway estimates. CHI'06 (pp. 454-459).

[2] Gallo, L., De Pietro, G., \& Marra, I. (2008). 3D interaction with volumetric medical data: experiencing the Wiimote. Proc. of the 1st ICST (p. 14).

[3] Lethbridge, T. C., Sim, S. E., \& Singer, J. (2005). Studying software engineers: Data collection techniques for software field studies. Empirical Software Engineering, 10(3), 311-341. Springer.

[4] Rogers, Y., \& Lindley, S. (2004). Collaborating around vertical and horizontal large interactive displays: which way is best? Interacting with Computers, 16(6), 1133-1152.

[5] Carrillo, a, Duerk, J. L., Lewin, J. S., \& Wilson, D. L. (2000). Semiautomatic 3-D image registration as applied to interventional MRI liver cancer treatment. IEEE transactions on medical imaging, 19(3), 175-185.

[6] Skounakis, E., Sakkalis, V., Marias, K., Banitsas, K., \& Graf, N. (2009). DoctorEye: A multifunctional open platform for fast annotation and visualization of tumors in medical images. IEEE EMBS 2009. 3759-62.

[7] Ure, J., Rakebrandt, F., Lloyd, S., \& Khanban, A. A. (2008). Usability, the Tri-Wizard Challenge: Recurring Scenarios in the Design of a HealthGrid Portal. HSI, 2008. Pages 298 - 305

[8] Derboven, J., Roeck, D. D., \& Verstraete, M. (2010). LowFidelity Prototyping for Multi-Touch Surfaces. Presented in the workshop Engineering Patterns for Multi-Touch Interfaces held in EICS 2010, 2nd edition, Berlin, Germany

[9] Keinonen,T. (2008).User-centered design and fundamental need. Proc. of the 5th Nordic conference on HCI (pp. 211-219).

[10] Hosseini-Khayat, A., Seyed, T., Burns, C., \& Maurer, F. (2011). Low-fidelity prototyping of gesture-based applications. Proceedings of the 3rd ACM SIGCHI (pp. 289-294).

[11] Evoluce multi-touch table. Retrieved from http://www.evoluce.com/en/hardware/multi-touch_table.php, accessed in March of 2012.

[12] Abdullah, N., Ngah, U. K., \& Aziz, S. A. (2011). Image classification of brain MRI using support vector machine. (IST), 2011 IEEE International Conference on (pp. 242-247).

[13] Boll, D. T., Duerk, J. L., \& Wilson, D. L. (2002). Image registration for interventional MRI-guided minimally invasive treatment of prostate cancer. Proc. of 24th EMBS 2nd joint, 1185 .

[14] Wobbrock, J. O., Morris, M. R., \& Wilson, A. D. (2009). Userdefined gestures for surface computing. Proceedings of CHI '09, 1083. New York, New York, USA

[15] Muto,W.,M.,Israelski, E.W. (2007). How new technologies can help create better UI's for medical devices. In Proceedings of HCI'07, Berlin, Heidelberg, 499-507.

[16] Windows Presentation Foundation, available at http://msdn.microsoft.com/en-us/library/ms754130.aspx, accessed December 2010 